01

\title{
Расчет коэффициента самораспыления при бомбардировке твердого тела ионами: компьютерное моделирование и теория
}

\author{
(С) А.И. Толмачев, ${ }^{1}$ Л. Форлано ${ }^{2}$ \\ ${ }^{1}$ Российский новый университет, \\ 105005 Москва, Россия \\ ${ }^{2}$ Universita della Calabria, \\ 87036 Cosenza, Italia \\ e-mail: tolmachev.alex@rambler.ru, forlano@vegachess.com
}

(Поступило в Редакцию 25 декабря 2017 г.)

Создана программа компьютерного моделирования для исследования самораспыления твердых тел под действием ионной бомбардировки. Программа основана на приближении парных столкновений, усеченном кулоновском потенциале, и модели локальных неупругих потерь, пропорциональных энергии. В результате моделирования вычислен коэффициент самораспыления в зависимости от порядкового номера и энергии ионов. Для проверки корректности моделирования атомных каскадов построена теория самораспыления для потенциала твердых сфер. Получено хорошее согласие результатов компьютерного моделирования с экспериментом и теорией в широком диапазоне энергий.

DOI: $10.21883 /$ JTF.2018.10.46492.2611

\section{Введение}

Исследование распыления твердых тел под действием ионной бомбардировки имеет важное значение при создании термоядерных установок и анализе поверхностных слоев вещества [1]. За последние десятилетия накоплено большое число экспериментальных данных по распылению, требующих своего объяснения [2]. Теоретическое рассмотрение проблемы затруднено тем, что при движении в мишени частицы тормозятся, и сечение рассеяния меняется с каждым новым столкновением. Основным инструментом исследования распыления в настоящее время являются программы компьютерного моделирования [2-4]. Большинство известных программ использует сложные атомные потенциалы и содержит большое число подгоночных параметров, зависящих от порядковых номеров и атомных масс ионов и атомов мишени. Значения параметров определяются из сопоставления результатов моделирования с экспериментом.

В настоящей работе разработана программа компьютерного моделирования PAOLA.SS, использующая при расчете упругих столкновений усеченный кулоновский потенциал и основанная на тех же принципах, что и программа PAOLA для расчета распределения отраженных ионов по пробегам в мишени [5]. Для усеченного кулоновского потенциала упругие столкновения частиц могут быть описаны аналитическими формулами, что позволяет избежать численного интегрирования, уменьшить время расчета и увеличить число пробных ионов. Число подгоночных параметров уменьшено до трех. Рассматривается случай самораспыления, при котором ионы и атомы мишени идентичны.

\section{1. Компьютерное моделирование}

Мы решаем следующую задачу. Ионы с энергией $E_{0}$ падают по нормали на поверхность полубесконечной мишени. Внутри мишени ионы сталкиваются с неподвижными атомами и выбивают их из положений равновесия. Выбитые атомы, в свою очередь, выбивают из положений равновесия новые атомы, и так далее - образуется каскад. Если энергия каскадного атома уменьшается до энергии отсечки $E_{\min }$, то этот атом считается остановившимся и больше не участвует в каскаде. Если же атом подходит к поверхности и выходит из мишени, то он считается распыленным и дает вклад в коэффициент распыления. Требуется найти зависимость коэффициента распыления от энергии и порядкового номера ионов. Следует заметить, что, поскольку ионы и атомы мишени неразличимы, полный коэффициент самораспыления представляет собой сумму коэффициента отражения рассеянных ионов $R_{N}$ и коэффициента распыления атомов отдачи $Y_{N}$.

Усеченный кулоновский потенциал имеет вид [6-8]

$$
U(r)=\frac{Z e}{4 \pi \varepsilon_{0}}\left(\frac{1}{r}-\frac{1}{r_{0}}\right) \quad \text { при } \quad r \leq r_{0},
$$

$U(r)=0$ при $r \geq r_{0}$, где $Z e-$ заряд атома, $r_{0}-$ радиус отсечки, $\varepsilon_{0}$ - электрическая постоянная. Для потенциала (1) вероятность образования атома отдачи с энергией от $T$ до $T+d T$ ионом энергии $E$ равна

$$
K(E, T) d T=\frac{\eta(1+\eta) d T}{E(\eta+T / E)^{2}},
$$

где $\eta=[4 \varepsilon(1+\varepsilon)]^{-1}$ - параметр экранирования, выраженный через приведенную энергию

$$
\varepsilon=2 \frac{E}{E_{a t}}\left(1+\frac{E}{E_{a t}}\right), \quad E_{a t}=\frac{Z^{2} e^{2}}{2 \pi \varepsilon_{0} r_{0}} .
$$


Сечение рассеяния (2) нормировано на единицу,

$$
\int_{0}^{E} K(E, T) d T=1,
$$

при малых энергиях $\varepsilon \ll 1$ описывает взаимодействие по закону твердых сфер, а при больших энергиях $\varepsilon \gg 1-$ кулоновское взаимодействие. Угол рассеяния $\Omega$ в лабораторной системе координат определяется соотношениeм

$$
\cos ^{2} \Omega=\frac{(1+\eta) b^{2}}{\eta+b^{2}}
$$

где $b-$ прицельный параметр, выраженный в единицах $r_{0}$. В результате упругого столкновения образуются две частицы с энергиями

$$
T_{1}=E \cos ^{2} \Omega, \quad T_{2}=E \sin ^{2} \Omega .
$$

Перед каждым столкновением частицы программа генерирует три случайных числа $R_{1}, R_{2}, R_{3}$ в интервале от 0 до 1 . Случайное число $R_{1}$ задает пробег частицы между столкновениями. Число $R_{2}=b^{2}$ определяет полярный угол рассеяния в уравнении (5) Число $R_{3}$ определяет азимутальный угол рассеяния [5].

Метод регистрации каскадных атомов в нашей программе отличается от метода, используемого в про-

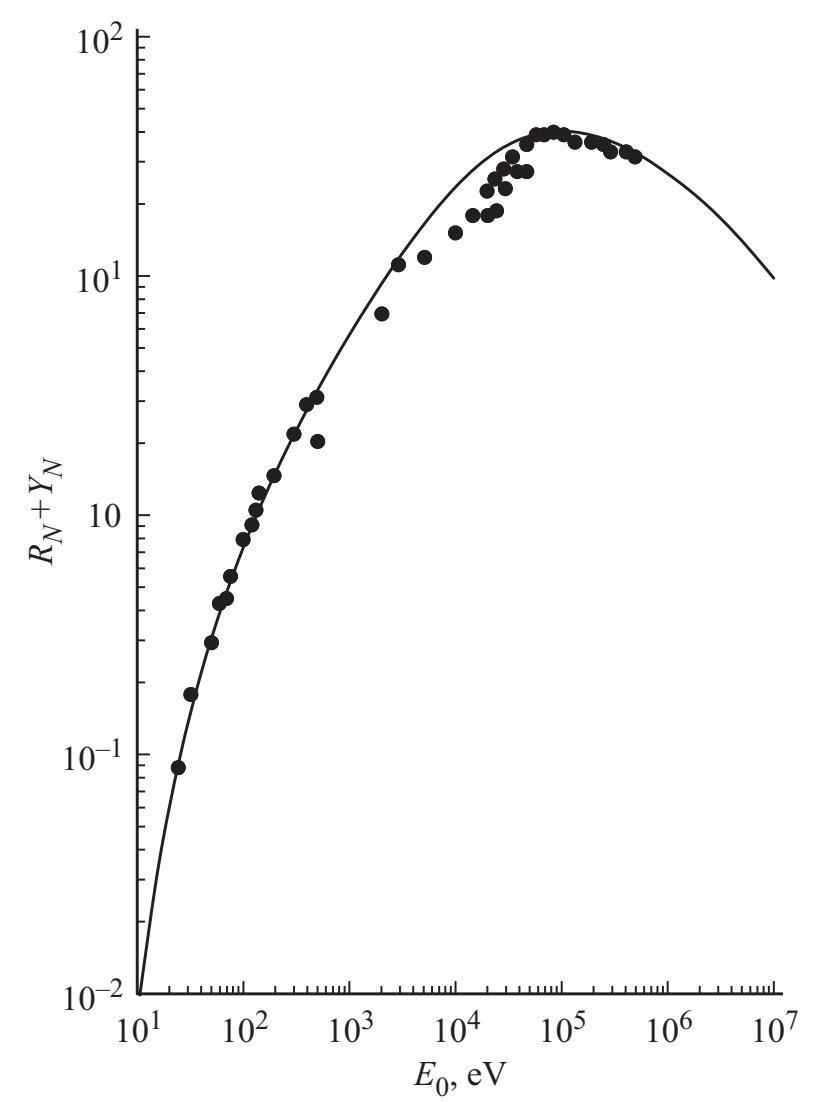

Рис. 1. Зависимость коэффициента самораспыления от энергии ионов для комбинации $\mathrm{Ag}-\mathrm{Ag}$. Маркеры - эксперимент [2], сплошная линия - расчеты по программе PAOLA.SS.

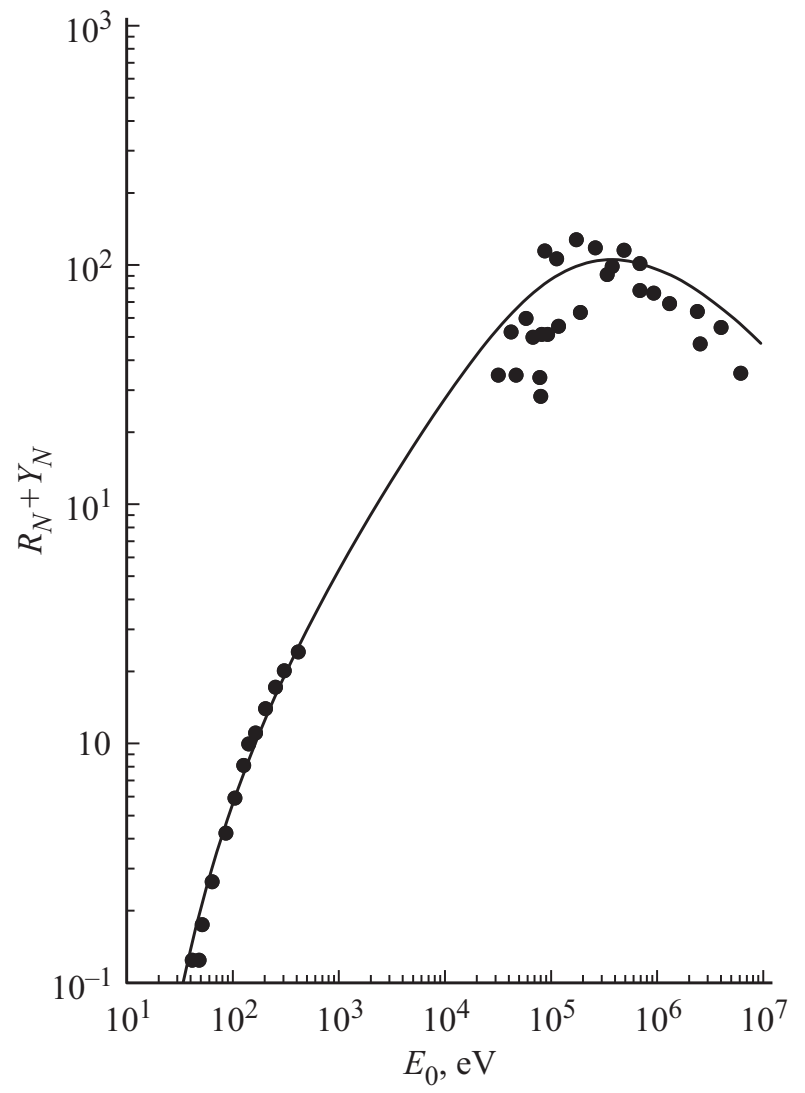

Рис. 2. Зависимость коэффициента самораспыления от энергии ионов для комбинации $\mathrm{Au}-\mathrm{Au}$. Маркеры - эксперимент [2], сплошная линия - расчеты по программе PAOLA.SS.

грамме TRIM.SP [3]. TRIM.SP проводит перену- мерацию атома отдачи после каждого нового столкновения. B программе PAOLA.SS каждому атому отдачи приписываются два фиксированных целых числа: порядковый номер и номер поколения каскада.

Алгоритм расчета каскада включает в себя нескольких этапов. 1) Падающий ион входит в мишень и выбивает из положений равновесия так называемые первично выбитые атомы (ПВА), которые образуют каскад первого поколения. Программа запоминает координаты и скорости всех ПВА. При дальнейшем движении ион либо останавливается, либо выходит из мишени и учитывается, как отраженный. 2) Рассматривается движение каждого ПВА и его столкновения с атомами мишени. В результате столкновений возникают вторично выбитые атомы (BBA), которые образуют каскад второго поколения. Программа запоминает координаты и скорости всех ВВА. В результате столкновений ПВА либо останавливаются, либо выходят из мишени и учитываются, как распыленные. 3) Аналогичным способом рассматривается каскады третьего, четвертого и следующих поколений. Каскад обрывается, если частица достигает поверхности мишени, или энергия частицы становится меньше энергии отсечки. 
Программа PAOLA.SS содержит три подгоночных параметра, зависящих от порядкового номера Первым параметром является энергия отсечки $E_{\min }$ которую обычно считают равной поверхностной энергии связи [3]. Вторым параметром является постоянная $C$, равная отношению радиуса отсечки к Боровскому радиусу: $C=r_{0} / r_{B}$. Третьим параметром является постоянная $D$ в законе неупругих потерь энергии. Мы считаем, что после каждого упругого столкновения частица с энергией $E$ теряет дополнительную энергию $\Delta E=D E$ за счет неупругих процессов. Сравнение с экспериментом показало, что локальные потери энергии дают лучшее согласие, чем непрерывные потери, а пропорциональность потерь энергии предпочтительнее, чем пропорциональность скорости. Постоянные $C$ и $D$ медленно уменьшаются с ростом $Z$. Отметим, что атомный потенциал ZBL, используемый в программе TRIM.SP, сам по себе уже содержит восемь подгоночных параметров.

На рис. 1 и 2 результаты моделирования сравниваются с экспериментальными данными для комбинаций $\mathrm{Ag}-\mathrm{Ag}$ и $\mathrm{Au}-\mathrm{Au}$ при энергиях ионов от $10 \mathrm{eV}$ до $10 \mathrm{MeV}$. Значения подгоночных параметров: $E_{\min }=1.0 \mathrm{eV}, C=9.0$, $D=0.37$ для $\mathrm{Ag}$ и $E_{\min }=1.5 \mathrm{eV}, C=6.6, D=0.32$ для $\mathrm{Au}$. Согласие можно считать хорошим с учетом того, что коэффициенты распыления меняются на три порядка.

\section{2. Теория}

Сечение рассеяния (2) не может быть использовано для теоретического анализа, так как угол рассеяния в уравнении (5) зависит от энергии и меняется от столкновения к столкновению. В большинстве теорий распыления [9-14] применяется степенное сечение

$$
K(E, T) d T=\frac{n T^{n-1}}{E^{n}}, \quad n>0,
$$

для которого зависимость угла рассеяния от прицельного параметра задается уравнением

$$
\cos ^{2} \Omega=1-\sqrt[n]{1-b^{2}}
$$

Если допустить, что показатель степени $n$ зависит от начальной энергии $E_{0}$ но не зависит от текущей энергии $E$ то формулы (7), (8) можно использовать для построения теории. В разд. 2 рассматривается случай $n=1$ соответствующий потенциалу твердых сфер. Неупругие потери энергии не учитываются.

Функция распределения каскадных частиц в мишени $f(x, \mu, u)$ зависит от нормированной глубины $x$ косинуса $\mu$ угла между скоростью частицы и внутренней нормалью к поверхности мишени и относительной энергии $u=E / E_{0}$. Уравнение переноса, усредненное по азимутальному углу, имеет вид

$$
\begin{aligned}
& \mu \frac{\partial f(x, \mu, u)}{\partial x}+f(x, \mu, u)=\int_{-1}^{1} d \mu^{\prime} \int_{0}^{1} d u^{\prime} \int_{0}^{\pi} \frac{d \varphi^{\prime}}{\pi} f\left(x, \mu^{\prime}, u^{\prime}\right) \\
& \times \sigma(\cos \Omega)\left[\delta\left(u^{\prime}-\frac{u}{\cos ^{2} \Omega}\right)+\delta\left(u^{\prime}-\frac{u}{\sin ^{2} \Omega}\right)\right], \quad(9)
\end{aligned}
$$

где угол $\Omega$ удовлетворяет соотношению

$$
\cos \Omega=\mu \mu^{\prime}+\sqrt{1-\mu^{2}} \sqrt{1-\mu^{\prime 2}} \cos \left(\varphi-\varphi^{\prime}\right)
$$

где $\varphi$ и $\varphi^{\prime}-$ азимутальные углы. Первое слагаемое в уравнении (9) определяет изменение числа частиц при движении между столкновениями. Второе и третье слагаемые представляют собой интеграл столкновений число частиц, уходящих и приходящих в состояние $(\mu, u)$ из состояния $\left(\mu^{\prime}, u^{\prime}\right)$. Дельта-функции в (9) указывают на то, что в результате столкновения возникают две частицы с энергиями, определяемыми уравнением (6).

Сечение рассеяния для упругого столкновения двух частиц равной массы, взаимодействующих по закону твердых сфер, имеет вид

$$
\sigma(\cos \Omega)=\cos \Omega+|\cos \Omega|
$$

Преобразование Меллина по энергии

$$
F(x, \mu)=\int_{0}^{1} u^{s-1} f(x, \mu, u) d u
$$

преобразует (9) в уравнение

$$
\begin{aligned}
& \mu \frac{\partial F(x, \mu)}{\partial x}+F(x, \mu)=\frac{2}{\pi} \int_{-1}^{1} F\left(x, \mu^{\prime}\right) d \mu^{\prime} \\
& \times \int_{0}^{\pi} \sigma(\cos \Omega) \cos ^{2 s} \Omega d \varphi^{\prime}
\end{aligned}
$$

где учтено, что слагаемые в квадратных скобках (9) после усреднения совпадают друг с другом.

Уравнение (13) представляет собой односкоростную задачу переноса с вероятностью рассеяния

$$
p\left(\mu, \mu^{\prime}\right)=\frac{2}{\pi} \int_{0}^{\pi} \sigma(\cos \Omega) \cos ^{2 s} \Omega d \varphi .
$$

Как показано в [15], решение задачи переноса можно свести к решению интегрального уравнения для функ- 
Зависимость коэффициента самораспыления от нормированной энергии ионов для потенциала твердых сфер. Сравнение компьютерного моделирования и теории

\begin{tabular}{c|c|c}
\hline \multirow{2}{*}{$\frac{E_{0}}{E_{\min }}$} & \multicolumn{2}{|c}{$R_{N}+Y_{N}$} \\
\cline { 2 - 3 } & paola.ss & Теория \\
\hline $10^{1}$ & $8.379 \cdot 10^{-2}$ & $8.390 \cdot 10^{-2}$ \\
$10^{2}$ & $3.868 \cdot 10^{+0}$ & $3.866 \cdot 10^{+0}$ \\
$10^{3}$ & $3.824 \cdot 10^{+1}$ & $3.829 \cdot 10^{+1}$ \\
$10^{4}$ & $3.085 \cdot 10^{+2}$ & $3.084 \cdot 10^{+2}$ \\
$10^{5}$ & $2.438 \cdot 10^{+3}$ & $2.442 \cdot 10^{+3}$ \\
$10^{6}$ & $1.971 \cdot 10^{+4}$ & $1.971 \cdot 10^{+4}$ \\
$10^{7}$ & $1.626 \cdot 10^{+5}$ & $1.627 \cdot 10^{+5}$
\end{tabular}

ции отражения $R\left(\mu, \mu_{0}\right)$,

$$
\begin{aligned}
& \left(\mu+\mu_{0}\right) R\left(\mu, \mu_{0}\right)=p\left(\mu,-\mu_{0}\right)+\mu \int_{0}^{1} p\left(\mu_{0}, \mu^{\prime}\right) R\left(\mu^{\prime}, \mu\right) d \mu^{\prime} \\
& +\mu_{0} \int_{0}^{1} p\left(\mu, \mu^{\prime}\right) R\left(\mu^{\prime}, \mu_{0}\right) d \mu^{\prime} \\
& +\mu \mu_{0} \int_{0}^{1} \int_{0}^{1} p\left(\mu^{\prime},-\mu^{\prime \prime}\right) R\left(\mu, \mu^{\prime}\right) R\left(\mu^{\prime \prime}, \mu_{0}\right) d \mu^{\prime} d \mu^{\prime \prime}
\end{aligned}
$$

где $\mu_{0}-$ косинус угла падения ионов.

Уравнение (15) было решено методом последовательных приближений [5], после чего интегрирование функции $R\left(\mu, \mu_{0}\right)$ по $\mu$ вычисление обратного преобразования Меллина и подстановка $\mu_{0}=1$ дали коэффициент самораспыления. В таблице приведены результаты сравнения теоретических результатов с результатами моделирования для различных отношений энергии ионов к энергии отсечки $E_{0} / E_{\min }$. При изменении энергии ионов на семь порядков, максимальная погрешность не превысила $0.2 \%$. Таблица подтверждает правильность методики расчета атомных каскадов, используемой в программе.

\section{Заключение}

Создана программа компьютерного моделирования для расчета коэффициента самораспыления в зависимости от сорта мишени и энергии ионов при нормальном падении на поверхность твердого тела. Программа содержит три параметра: энергию отсечки, параметр упругих столкновений и параметр неупругих потерь энергии. Расчеты показали, что зависимость коэффициента самораспыления от энергии имеет куполообразную форму с максимумом в районе $10^{5}-10^{6} \mathrm{eV}$. Результаты моделирования хорошо согласуются с экспериментальными данными для комбинаций $\mathrm{Ag}-\mathrm{Ag}$ и $\mathrm{Au}-\mathrm{Au}$ при энергиях ионов от $10 \mathrm{eV}$ до $10 \mathrm{MeV}$ и коэффициентах распыления от 0.1 до 100 .

Для проверки корректности программного кода при расчете атомных каскадов построена теория самораспыления для случая взаимодействия частиц по закону твердых сфер. Расхождение между моделированием и теорией оказалось очень малым и вряд ли может быть уменьшено в рамках методики Монте-Карло. Результаты теории могут служить для тестирования вновь создаваемых программ, моделирующих явление распыления.

\section{Список литературы}

[1] Борисов А.М., Машкова Е.С. Физические основы ионнолучевых технологий. II. Распыление поверхности твердых тел. М.: МАКС Пресс, 2013. 196 с.

[2] Behrish R., Eckstein W. (editors). Sputtering by Particle Bombardment. Berlin: Springer-Verlag, 2007. 470 p.

[3] Eckstein W. Computer Simulation of Ion - Solid Interactions. Berlin: Springer-Verlag, 1991. 296 p.

[4] Ziegler J.F., Ziegler M.D., Biersack J.P. The Stopping and Range of Ions in Matter. Version SRIM-2013.

[5] Толмачев А.И., Форлано Л. // ЖТФ. 2017. Т. 87. Вып. 7. C. $973-978$.

[6] Leibfried G., Oen O.S. // J. Appl. Phys. 1962. Vol. 33. N 7. P. 2257-2262.

[7] Толмачев А.И. // Поверхность. 1992. Вып. 8. С. 27-29.

[8] Толмачев А.И. // Поверхность. 1993. Вып. 12. С. 9-16.

[9] Sigmund P. // Phys. Rev. 1969. Vol. 184. N 2. P. 383-416.

[10] Sanders J.B., Roosendaal H.E. // Radiation Effects. 1980. Vol. 52. P. 137-144.

[11] Falcone G. // La Rivista del Nuovo Cimento. 1990. Vol. 13. N 1. P. $1-52$.

[12] Tolmachev A.I. // Nuclear Instruments and Methods. 1993. Vol. B83. P. 479-481.

[13] Tolmachev A.I. // Nuclear Instruments and Methods. 1994. Vol. B93. P. 415-420.

[14] Барат А.А., Манухин В.В. // ЖТФ. 2009. Т. 79. Вып. 2. C. 117-124.

[15] Chandrasekhar S. Radiative Transfer. Oxford: Clarendon Press, 1950. 393 p. 